\title{
The use of tannins in the local treatment of burn wounds - a pilot study
}

\author{
L Chokotho, E van Hasselt \\ Department of Surgery, College of Medicine University of Malawi
}

Twenty patients aged 3 years and above with fresh partial thickness burns of less than $20 \%$ total body surface area were randomly assigned to local treatment of the burn wound either with a solution containing tannins (tannin group), or one of the other commonly used topical agents, such as honey and ghee, silver sulfadiazine, etc (the other group). The effects on serum transaminase and alkaline phosphatase, the incidence and type of wound infection, the quality of the eschar, the formation of scar tissue, adverse effects, the alleviation of pain and possible adverse reactions were studied in both treatment groups. Surprisingly initial assessment of the burn wound depth proved more difficult than anticipated even in the hands of the more experienced health worker. Liver function tests were elevated in both the tannin ( 6 patients) and the other group ( 7 patients). Bacterial colonization of the wounds was equally distributed in both groups, although Staphylococcus aureus was found more often in the other group ( 9 versus 1$)$. The quality of the eschar was better in the tannin group ( 6 patients had a supple eschar versus 3 in the other group). The most important observations were that no adverse reactions were encountered and that in the tannin group only one patient developed a hypertrophic scar compared to 5 patients in the other group. It proved difficult to quantify alleviation of pain especially in the children.

\section{Introduction}

A burn injury is a distressing and challenging condition which is common in Malawi. A whole array of remedies has served in the local treatment of burns through the ages1. The use of dressings with antibacterial topical agents in the local treatment of burn wounds is the current standard. It is a time consuming and often painful method. A number of the topical agents used are either expensive or difficult to acquire.

Davidson 2 in 1925 started to treat burn wounds with tannins on the assumption that it reduced the systemic reaction due to the absorption of toxic substances from the burned skin. Tannins are widely distributed in plants and are divided in two types, hydrolysable and condensed tannins. The latter are found in tea plants3. More recent research has found that tannin has the property to form complexes with several biomolecules and therefore might be efficacious in precipitating poisonous materials in burned tissue1. Tannin also leads to improved wound healing and reduced scar tissue formation by inhibition of the formation and removal of reactive oxygen substances. Additional advantages of tannins include: relief of pain, limitation of secondary infection, prevention of loss of plasma and promotion of prolific epithelialisation 4,5 .

The use of tannin in the treatment of burn wounds was abandoned in the mid forties due to the availability of topical antibacterial agents and the associated hepatotoxic effects observed in both patients and experimental animals4,5. However critical evaluation of early literature and experimental observation has shown that the hepatotoxic effects are not related to tannin but are due to the burn syndrome itself 6 . In addition use of tannin results in formation of supple eschar. The use of tannin as adjuvant therapy for burn wounds therefore has regained interest.

Because of the availability of tea and its by-products and its low cost we conducted a pilot study in the use of tannins in the topical treatment of the burn wound and compared the outcome of this treatment with the currently used agents.

\section{Methods}

The following parameters were studied: effect on serum transaminase and alkaline phosphatase, incidence and type of wound infection, alleviation of pain, quality of eschar, scar tis- sue formation 3 months post burn and adverse reactions. Burn patients were recruited who met the following criteria: presentation on the day of the burn; 3 years of age or older; total body surface area (TBSA) burnt of less than $20 \%$ using Lund and Browder chart; and partial thickness burns.

Informed consent was obtained from the patient or guardian. The patients were randomly assigned to treatment with either a fresh solution of tannins twice daily or one of the commonly used topical agents such as honey and ghee, silver sulfadiazine, silver nitrate, Eusol and paraffin and Zinc oxide. The tannin solution was prepared by mixing 10 grams of ground tea stalks with $100 \mathrm{cc}$ of boiling water and left for 5 minutes. The solution was then filtered and allowed to cool down and subsequently used. The strength of tannin obtained this way proved to be the correct one after being tested in a laboratory in Utrecht.

Blood for liver function tests (LFT's) and wound swabs were taken at weekly intervals. Pain charts were used, (visual ones for children). Pictures with a digital camera were made to record wound appearances at weekly intervals, at discharge and 3 months post burn. A separate form to report adverse drug effects was included in the study file.

\section{Results}

Ninety percent of the patients were less than 10years old. Hot water was the commonest cause of burn wounds. Most patients sustained partial thickness burn wounds (table 1). LFT's were elevated in both groups. Almost all wounds were colonised by bacteria with staphylococcus as the most common bacteria cultured (table 2). Supple eschar formed mostly in the tannin group and only one patient in the tannin group developed a hypertrophic scar (table 3).

\section{Discussion}

Hot water was the commonest cause of burn wounds. This is as expected in societies where cooking takes place at ground level and children are allowed near it unguarded or being looked after by a young sibling. The initial assessment of depth was more difficult than anticipated since some patients who were recruited as partial thickness ended up being full thickness. Intensive laboratory testing was too difficult within the existing staffing 
levels. Elevation of LFT's in both groups suggests that this was due to the burn syndrome itself. Quantification of the level of pain was difficult in children as all pointed towards "hurts terribly".

This study was not blinded. However, results of this pilot study suggest that the use of tannins may provide benefit by reducing colonisation of $\mathrm{S}$. aureus with better quality of healing and at the same time not increasing toxicity. There was only one case of hypertrophic scar formation in the tannin group. No adverse reactions were encountered in either treatment group. The use of tannin in the treatment of partial thickness burn wounds could be advantageous and cheap in places where tea and its by-products are easily available. A larger study population is required to see whether these potential benefits are clinically significant.

References

1. Health Management Publications, Inc. Wounds 13(4): $144-$ 158, 2001. http://www.medscape.com

2. Davidson EC. Tannic acid in the treatment of burns. Surg Gynecol Obstet 1925; 41:202-21

3. Haslam E. Practical Polyphenolics: From Structure to Molecular Recognition and Physiological Action.Cambridge, UK: Cambridge University Press, 1998:10-83

4. Halkes SB A et al, The use tannic acid in the local treatment of burn wounds: intriguing old and new perspectives. Wounds 13(4):144-158, 2001

5. Hupkens P et al, Tannic acid as a topical agent in burns: historical considerations and implications for new developments. Burns 21(1) 57-61, 1995

6. Kreis RW et al, Tannin Ointment. Clinical pilot trial report. Burns research institute 1992

Table 1: Patient Demography, Cause and Depth of the Burn Wound

\begin{tabular}{lccc}
\hline & & TANNIN & OTHER \\
\hline Sex & Male & 8 & 6 \\
& Female & 2 & 4 \\
Age & Range & $3-38$ & $4-50$ \\
& $<10$ years & $10(50 \%)$ & $8(40 \%)$ \\
Cause & Water & 8 & 8 \\
& Fire & 2 & 2 \\
Depth & Superficial PT & 7 & 6 \\
& Deep PT & 2 & 1 \\
& Mixed & 1 & 3 \\
& & & \\
& & & \\
& & & \\
& & & \\
& & & \\
& & &
\end{tabular}

Table 2: Laboratory investigations

\begin{tabular}{cccc}
\hline & & TANNIN & O'THER \\
\hline LFT's & Raised & 6 & 7 \\
& Normal & 3 & 2 \\
& N/A & 1 & 1 \\
Bacterial growth & Yes & 5 & 9 \\
& No & 3 & 0 \\
& N/A & 2 & 1 \\
Staphylococcus & & 1 & 9 \\
\hline
\end{tabular}

Table 3: Comparison of eschar quality and outcome of scar

\begin{tabular}{cccc}
\hline & & TANNIN & OTHER \\
\hline \multirow{3}{*}{ ESCHAR } & Supple & 6 & 3 \\
& Hard & 1 & 4 \\
& N/A & 3 & 3 \\
\multirow{3}{*}{ HYPERTROPHY } & No & 1 & 5 \\
& N/A & 4 & 1 \\
& & 4 & 4 \\
\hline
\end{tabular}

